\title{
Virtual reality in marketing communication - the impact on the message, technology and offer perception - empirical study ${ }^{1}$
}

\author{
Filip Grudzewski², Marcin Awdziej', Grzegorz Mazurek², \\ Katarzyna Piotrowska ${ }^{3}$
}

\begin{abstract}
VR technology is an emerging IT innovation that greatly affects consumer behaviour and consumer perception of products. The aim of this study is to examine how the virtual reality phenomenon can be used as a marketing communication tool and how its usage affects the reception of individual components of a marketing message. The research conducted examined the possible impact of virtual reality on message perception and attitude towards particular offers. Additionally the authors wanted to find out whether there was a relationship between the use of virtual reality and the acceptance of new technologies in marketing communication. To verify the stated hypotheses empirical research was conducted involving an experiment with 150 observations of respondents taking advantage of three different marketing communication tools including: VR presentation with Oculus Rift hardware, video and printed advertisements. The results obtained reveal that VR technology positively and significantly impacts the reception of the offer, the technology involved and the presentation itself.
\end{abstract}

Keywords: virtual reality, VR, technology, marketing communication, advertising, virtualization.

JEL codes: M31, M37.

\section{Introduction}

Virtual Reality is a computer based technology that makes it possible to simulate a real environment in which the user can experience the feeling of being present (Serrano, Botella, Baños and Alcañiz, 2013). Virtual reality is often

\footnotetext{
${ }^{1}$ Article received 18 December 2017, accepted 24 April 2018.

${ }^{2}$ Koźmiński University, Department of Marketing, ul. Jagiellońska 57/59, 03-301 Warsaw, Poland,gmazurek@kozminski.edu.pl.

${ }^{3}$ Koźmiński University, Department of Quantitative Methods \& Information Technology, ul. Jagiellońska 57/59, 03-301 Warsaw, Poland.
} 
characterized as an environment created by a computer or other media, an environment in which the user feels present (Biocca, 1992a, p. 5). Coates (1992) explains that Virtual Reality involves electronic simulations of environments experienced through head mounted eye goggles and wired clothing enabling the end user to interact in realistic three-dimensional situations. The number of VR users may increase rapidly with possibly up to 171 million active users in 2018, including 28 million users willing to pay for the content offered (The Farm 51, 2015, p. 11). This is all possible because of a massive growth in the popularity of VR products, which let people escape from the physical reality and dive deep into a virtual one, along with the high applicability of VR systems in such industries as: video entertainment, live events, education, retail, real estate, military, healthcare and engineering (Benefield, Rutherford, \& Allen, 2012; Hassouneh \& Brengman, 2015). It is expected that this trend will spread faster than was the case with the Internet and smartphones, especially since in order to consume basic VR content consumers do not need to invest in very expensive hardware they just have to add accessories that will change their smartphones into VR devices (Barnes, 2016).

The consumer drive towards new technologies "(...) allows them to see the surrounding world in another dimension and to experience things that are not accessible in real life or even not yet created" (Mazuryk \& Gervautz, 1996). Technology has become a package that makes it possible to deliver information to the end user (Kaplan \& Mazurek, 2018). The amount of existing software is expanding rapidly as are the opportunities for companies to enter this lucrative market and to use new solutions as great marketing tools to communicate their advertising to users (Scatena, Russo, \& Riva, 2016, p. 212).

Virtual reality provides a gateway for marketeers to reach consumers in new ways (Van Kerrebroeck, Brengman, \& Willems, 2017a). A company that takes advantage of VR technology can benefit from major improvements in their marketing communication as well as in the level of their customers' knowledge of the products on offer (Huang, Backman, K.F., Backman, S.J., \& Chang, 2015; Van Kerrebroeck et al., 2017a). Moreover consumers gain value from VR technology, which enhances their lives, and so businesses and advertisers need to reach them by these means in order to identify new opportunities of providing consumers with even more value (Jung, Dieck, Lee, \& Chung, 2016).

The research aim was to investigate whether the type of promotion material influenced the perception of a given offer among the target audience. Secondly the authors also wished to find out whether the type of the promotion material involved influenced the reception of the technology and the message communicated itself. The paper is divided into four sections. The first section explains the theoretical background. The second outlines the methodology applied. The third section presents the results and discussion. The paper ends with conclusions. 


\section{Virtual reality - the concept}

The concept of virtual reality is a bit of a conundrum (Steuer, 1992), offering many equivalent definitions (e.g. virtual environments, synthetic experience, virtual worlds, artificial worlds, artificial reality). Virtual reality is a medium composed of interactive computer simulations that sense the participant's position and actions and replace or augment the feedback to one or more senses, offering the participant a feeling of being mentally immersed or present in the simulation (a virtual world) (Sherman, 2003, p. 13). Virtual reality induces targeted behaviour by using artificial sensory stimulation, while the organism has little or no awareness of the interference (La Valle, 2017, p. 1). Mazuryk and Gervautz (1996) describe VR as "an interactive and immersive (...) experience in a simulated (...) world". The highlight the fact that given the greater interest in computer graphics the boundary between $3 \mathrm{D}$ computer graphics and virtual reality is blending and thus it is very important to determine appropriate definitions of VR technology components. Two terms that are very important in the VR dictionary are also mentioned. The first of them is telepresence, defined as the experience of presence in an environment by means of a communication medium (Steuer, 1992, p. 79). It happens when an operator manipulates the user remotely from a distance (operator's room) and at the same time receives a sensory feedback that lets them feel just as if they are in the place where the action occurs. Telepresence is considered an important construct in virtual reality research where two main perspectives dominate: a psychological and a technological (Yin, Cicchirillo, \& Drumwright, 2012). Various studies have found that presence influences the perceived product knowledge, brand attributes (Grigorovici \& Constantin, 2004; Hopkins, Raymond, \& Mitra, 2004), recall and recognition (Keng \& Lin, 2006), attitudes towards advertisements (Hopkins et al., 2004) and purchase intentions. Generating a sense of presence depends on the media involved, user characteristics (perceptions of interactivity and vividness) and individual attention to media stimuli (Lessiter, Freeman, Keogh, \& Davidoff, 2001).

Mazuryk and Gervautz (1996) have also pointed out how VR experience is actually delivered to the user. In theory a computer should generate multiple-sensory impressions that affect all of the user's senses on various levels so that they can experience a full immersion in a realistically responsive virtual reality. Based on the level of immersion the authors name three types of VR systems. The first is Desktop VR, also referred to as a Window on the World, which lets users only watch the content offered using regularly displayed images. Fish Tank VR is an evolution of Desktop VR. It takes advantage of the motion parallax effect to enhance the VR impressions because it supports head tracking; however there is no sensory feedback for the user and the image is still displayed on the screen. The third level is based on immersive systems. This technology is implemented in the most advanced VR devices and 
includes head-mounted display (HMD) to provide the best possible view to the user - a first-person perspective and reacts to changes in his position or orientation. The whole simulation can be also enhanced further by sound or haptic and sensory stimuli.

The specificity of the VR technology is that in combining hardware and software together it can create a reality where people can find various types of possibilities. From creating a whole new entertainment segment (such as movies, games, free time apps), improving whole navigation processes (virtual $360^{\circ}$ maps of routes and places - such as public spaces, buildings, subway stations, guides), through medical application (virtual surgery training or a 3D image of the inner body shown to explain medical cases), cultural and science application (museum exhibitions, virtual learning) ending with the segment for children (bedtime stories, discovering the world apps), etc.

\section{Virtual Reality in marketing communication}

Digitalization of communications is an increasing phenomenon, reflected in the way of planning and executing marketing communication (Mazurek, 2011a). Efficient utilization of marketing communications requires not only a very good knowledge of social phenomena and market processes but also familiarity with the instruments and technologies of information communication. Both marketing theoreticians and practitioners agree that the key condition for marketing communications to be effective is integration of all the means thereof. All the undertaken communication activities need to be consistent in terms of their content and coordinated in time and space. Companies utilising the methods and tools of modern marketing communications are able to interact quickly with consumers (Hajduk, 2016).

Virtual reality is a relatively new medium that offers new opportunities for content communication. The qualities of virtual reality make it able to manipulate the sense of time and space, to be interactive, and make the user 'control' their experience. According to Biocca (1992b), there are many research implications of VR on communications, including studies on: the diffusion of virtual reality technology, communication design and cognition or interpersonal communication and cooperative work. All this offers an incredible opportunity to establish a dynamic relationship between the audience and the medium where interactivity and responsivity play a crucial part (Mazurek, $2011 b)$. An ideal situation is one that involves the creation of a high-quality interface - based on platforms connecting devices, or rather whole realities - so that the user does not see the difference between their own and the virtual world (Barnes, 2016).

Before the era of the growth of the introduction of new technologies and virtualization processes (Mazurek, 2012) it was quite easy to predict the tools and 
media needed to communicate with several groups of stakeholders effectively (i.e. to increase brand awareness, communicate sales promotion and proceed with personal selling for business-to-business markets). With time technology (progression in the advancement and spread of the Internet) has made it possible to reach certain customers directly faster and more straightforwardly than ever which has brought about substantial changes in the style and nature of marketing communication.

Virtual Reality (VR) is considered to be one of the most promising technological innovations in business (Gartner, 2016). As it has become more accessible to consumers at affordable prices it has caught the attention of marketeers as a new way to advertise products and brands (Adams, 2016). Virtual Reality is applied in tourism industry (making it possible to visit various destinations), experience marketing (allowing the experience of lifestyles associated with particular brands) and various product tests (e.g. test drives of cars) (Scott, 2016; Mandelbaum, 2015). Despite the growing interest in the application of Virtual Reality in real estate marketing there are not many studies that have investigated the effectiveness of this medium in this specific context. However a study conducted by Benefield et al. (2012) indicated that increasing the visibility of a property through a mix of media, such as open houses and virtual tours, generated higher selling prices.

Retailers such as Carrefour and Alibaba explore opportunities for VR in online shopping and product inspections (Zheng, 2016). Over the last years advertising has evolved along with technology, embracing such innovations as $3 \mathrm{D}$ product presentations and $360^{\circ}$ rotation. Compared with the traditional media these allow higher levels of interactivity and vividness resulting in visual richness (Choi and Taylor, 2014; Van Kerrebroeck et al., 2017b). Vividness, or imagery richness, is considered to be an important factor in marketing communications translating into more realistic product presentations. Cheng, M.H. Chieng, and W.H. Chieng (2014) suggest that imagery richness can be affected by sound or animations. Various studies in marketing literature demonstrate that more vivid imagery results in more positive attitudes among consumers (Van Kerrebroeck et al., 2017b). Existing studies point consistently to the benefits of application of VR technology to informational marketing communications - that is, to presentation of functional, objective product attributes (Choi and Taylor, 2014). When it comes to emotional, i.e. hedonic benefits associated with products or brands literature is almost non-existent, with a notable exception of a study by Van Kerrebroeck et al. (2017b). These authors found that VR generates higher perceptions of vividness and presence compared to a regular, two-dimensional video (Van Kerrebroeck et al., 2017b). The vividness of VR presentation positively affects consumers' attitudes towards advertisements and brands and stimulates purchase intentions. VR technology improves the communication process and, at the same time, offers a better perception of the marketing message as well as of the advertised products. Thus: 
H1: The form of the offer's presentation influences its perception.

As the product presentation perception is not a unidimensional experience it was decided to investigate the relationship between the form of the presentation and the three aspects of the presentation's perception.

H1a: The form of the presentation influences the perceived attractiveness of the offer presented (in this case: an apartment).

H1b: The form of the presentation itself influences its reception.

H1c: The form of the presentation influences the attitudes towards the use of in-house technology.

\section{Methodology}

Respondents were recruited from a group of clients of coffee houses where the experiment was conducted. A total number of 150 usable responses was collected from 75 men and 75 women with a mean age of $36.41(S D=8.86)$. The participants were randomly assigned to three groups (picture, video, and virtual reality space -50 persons each ( 25 females and 25 males in each group)). It was decided to control the study for gender, as there are gender differences in visual perceptions and attitudes towards technology. (Schroeder, 2010; Sax, 2006). Therefore in each of the groups the number of males and females was equal (i.e. 25).

The experiment was conducted out of peak hours, before noon, to ensure that the respondents would be least distracted by their surroundings. The respondents were approached by the researchers and asked to participate in the experiment. Once agreed the subject and the purpose of the study were explained to them and all the required details provided. Then the respondents drew lots from a non-transparent bag which included 150 cards (50 containing a description of an apartment with a picture, 50 with a description and a video and 50 with a description and a Virtual Reality presentation in Oculus). ${ }^{4}$ The draw was made without returning the cards into the bag. The respondents were presented the apartment promotional materials in pictures, in a video, or as a Virtual Reality presentation in which they had an opportunity to "walk

\footnotetext{
${ }^{4}$ Photos: 5 photos, resolution $1928 \times 1092$, printed on A4 size paper sheets, presenting the apartment from simulation. They were presented as marketing material (a leaflet). Video: MPEG-4, $1280 \times 720$, length $1: 20$, ordinary video displayed on a 15.4-inch laptop. It was the material obtained from the company, it was not a 360 degree video because it was desired to maintain an atmosphere of an ordinary advertising video (the simulation was in 360 degree, so, the overlap was not necessary). Oculus presentation: Simulation was created by TRUSENSE as one of their projects. It was a fully interactive simulation of the apartment, the user could move around the apartment in two ways - for a longer distance, there was a teleportation, and for a short distance, the user could move around on foot (an Oculus camera tracks movement of the user enabling movement in the simulation).
} 
around the place" using VR goggles on their eyes (Oculus Rift). The length of exposure to the promotional material in any form was estimated at around 1 minute 20 seconds - as long as the length of the video. It was to ensure the same time exposure to each kind of material without repeating it. Also, in the case of the video and the Oculus presentation, the sound was turned off as the focus of the study was on the visual aspect of presentation.

After the presentation of the material each respondent filled in a questionnaire which consisted of 18 closed questions related to their perception of the apartment presented, an evaluation of the presentation itself and the attitude towards the new technologies featured in the apartment featured ${ }^{5}$. The respondents answered every question using a scale from 1 to 10 , where 1 meant "Definitely not", which was the scale's minimum, and 10 meant "definitely yes". To see whether the questions designed to measure the three aspects of the offer could be grouped into composite indexes, the most commonly used measure of the scale's internal reliability was Cronbach's alpha and mean inter-item correlations were also calculated (Cortina 1993; George \& Mallery 2003; Allameh, Esfahani, \& Nikbakht, 2017). The Cronbach's alpha for each of the scales was above 0.75 (as for the apartment's attractiveness, it was $0.871,0.954$ for the presentation assessment scale, and 0.891 for the scale measuring the attitude towards the featured technology) ${ }^{6}$, and the average inter-item correlation ranged from 0.529 (for the apartment's attractiveness scale) to 0.786 (for the presentation assessment scale). The high values of the scales' internal reliability made it possible to calculate the composite indices for every group of questions. ${ }^{7}$ For each of the scales, 1 meant the lowest score, and 10 - the highest.

As the participants were randomly assigned to groups (picture, video, Oculus) an analysis of variance (ANOVA) was performed to explore the relationship between the variables with the F-test calculated to test for the significance of differences between the groups (Saunders, Lewis, \& Thornhill, 2009; Kirk, 2013). However due to the fact that the distribution of the dependent variable could not be considered close to normal in every group (i.e. the absolute values of skewness and kurtosis in several cases were higher than 1 (Hair, Black, Babin, \& Anderson, 2010), the Kruskal-Wallis rank test (Conover \& Iman, 1981) was applied as well to check whether the ANOVA results were not caused by data distribution anomalies. To assess the effect size, an eta squared $\left(\eta^{2}\right)$ estimate for Kruskal-Wallis H-test was calculated (Tomczak, M. $\&$ Tomczak E., 2014) and to test for the significance of the differences for every pair of the groups compared (i.e. picture-video, video-oculus, oculus-picture), a non-parametric post hoc test (the Dunn's post hoc test adjusted by the

\footnotetext{
${ }^{5}$ Additional visual materials may be obtainable from the authors upon request.

${ }^{6}$ Detailed information regarding the items included in the scales is presented in Appendix 1.

${ }^{7}$ In order to obtain composite indices, the arithmetical means of the answers included in each of the scales were calculated.
} 
Bonferroni correction for multiple tests) ${ }^{8}$ was used. All the statistical analyses were carried out using SPSS 24.0 software.

\section{Results and discussion}

As shown in Table 1 the $\mathrm{F}$ tests as well as the $\mathrm{H}$ tests for all three dependent variables were statistically significant which means that all the formulated hypotheses (H1, H2 and $\mathrm{H} 3$ ) are confirmed.

Considering all the composite indices the means and medians are the lowest for the PICTURE exposition, higher in the case of the VIDEO and the highest in the case of the OCULUS VR system usage and the differences between groups are the largest for the presentation perception (Table 1 and Figure 1).

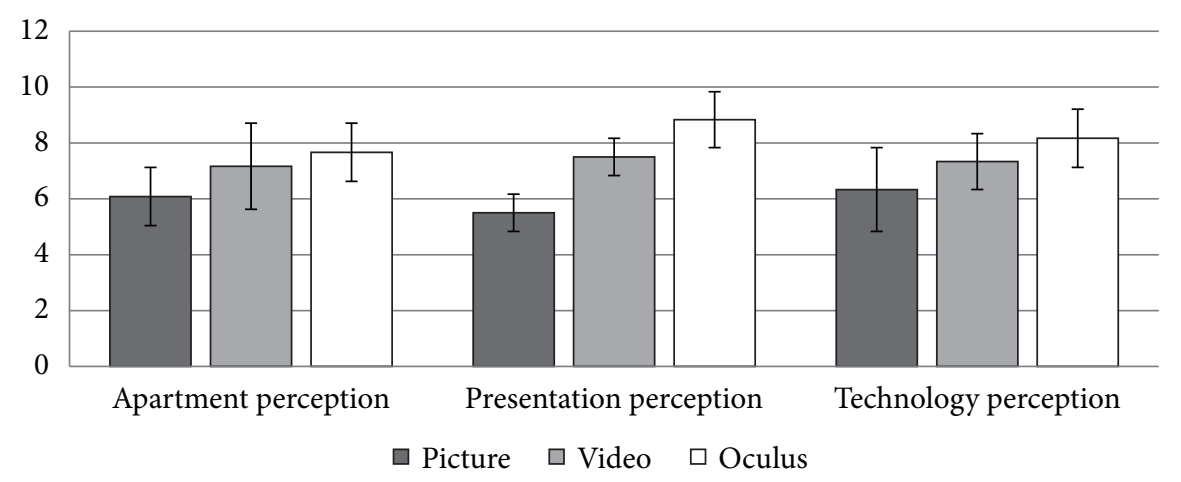

Figure 1. Median values of the dependent variables in the groups compared (error bars show the interquartile ranges)

Source: own research.

The Eta squared estimates show that the variance of the apartment perception is explained by the type of means in $36 \%$, presentation perception in $72 \%$, and technology perception in $49 \%$. This means that the effect was large in every case (Cohen 1988) ${ }^{9}$ and that the dependency between the type of the presentation material and the respondents' evaluation was the strongest in terms of the perception of the presentation itself.

The perception of the relationship between the applied technology and the means of presentation is also quite high (between apartment and presentation). It is similar to the occurrence of the halo effect that influenced the positive

\footnotetext{
${ }^{8}$ This is the only non-parametric post hoc test available in SPSS software.

${ }^{9}$ According to Cohen (1988), large effects start from eta squared $=0.14$ for the analysis of variance.
} 


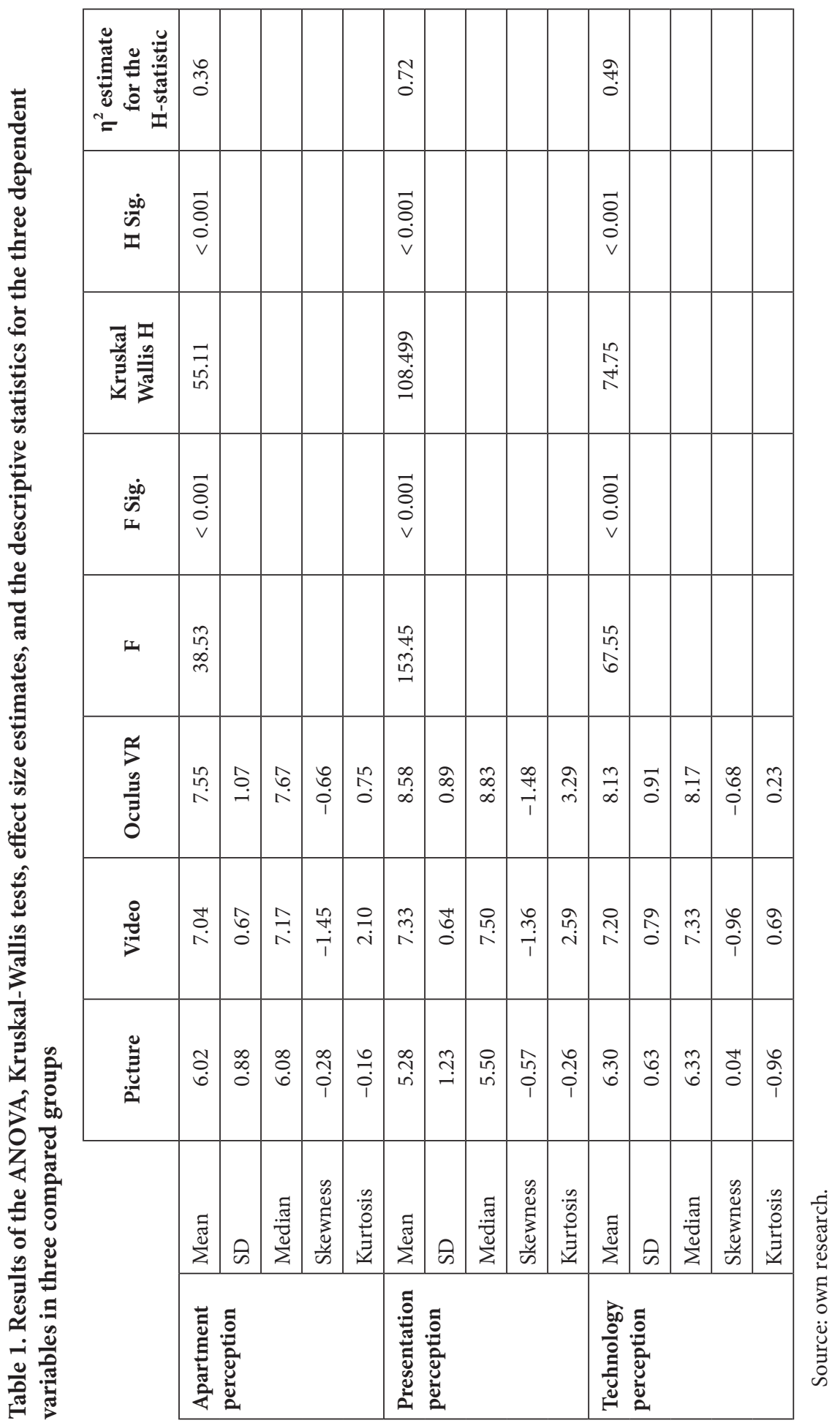


perception of the technology because of the usage of another new technology (Oculus VR system used to present the offer).

Table 2. Results of multiple pairwise comparisons (Mann-Whitney $U$ adjusted by the Bonferroni correction for multiple tests)

\begin{tabular}{|l|c|c|c|c|c|c|}
\cline { 2 - 7 } \multicolumn{1}{c|}{} & \multicolumn{2}{c|}{ Apartment perception } & \multicolumn{2}{c|}{$\begin{array}{c}\text { Presentation } \\
\text { perception }\end{array}$} & \multicolumn{2}{c|}{$\begin{array}{c}\text { Technology } \\
\text { perception }\end{array}$} \\
\cline { 2 - 7 } \multicolumn{1}{c|}{} & $\begin{array}{c}\text { Test } \\
\text { Statistic }\end{array}$ & $\begin{array}{c}\text { Adjusted } \\
\text { Significance }\end{array}$ & $\begin{array}{c}\text { Test } \\
\text { Statistic }\end{array}$ & $\begin{array}{c}\text { Adjusted } \\
\text { Significance }\end{array}$ & $\begin{array}{c}\text { Test } \\
\text { Statistic }\end{array}$ & $\begin{array}{c}\text { Adjusted } \\
\text { Significance }\end{array}$ \\
\hline Picture-Video & -42.22 & $<0.001$ & -48.97 & $<0.001$ & -39.58 & $<0.001$ \\
\hline Picture-Oculus & -63.20 & $<0.001$ & -90.32 & $<0.001$ & -74.96 & $<0.001$ \\
\hline Video-Oculus & -20.98 & 0.047 & -41.35 & $<0.001$ & -35.38 & $<0.001$ \\
\hline
\end{tabular}

Source: own research.

The pairwise comparisons (Table 2) showed that in each of the three pairs (Picture-Video, Video-Oculus, Oculus-Picture) the differences in the evaluation were statistically significant for each of the dependent variables. In all cases, apart from the Video-Oculus comparison for apartment perception $(p<0.05)$, the test was significant at $\alpha=0.001$ level.

The results of the research show that new technologies enhance marketing communication to a significant extent, translating into a better reception of marketing messages among potential customers. VR technology improves the communication process and, at the same time, offers a better perception of the marketing message as well as of the advertised products. The study has proven that VR technology is ahead of the current means of marketing communication. H1: The form of the offer's presentation influences its perception.

The research has confirmed that there is a significant relationship between the means of presentation and the presentation's perception among the respondents. As shown by the comparison between the most traditional means (pictures) and the most modern solution (Oculus VR system).

H1a: The form of the presentation influences the perceived attractiveness of the presented offer (in this case: an apartment).

The apartments presented were highest evaluated by the respondents who were shown the offer through the Oculus VR tool. The video presentation did a little worse and the worst result was obtained in the case of communication through pictures. It means that Oculus offers a better user experience and increases the attractiveness of the offer presented.

H1b: The form of the presentation itself influences its reception.

Here Oculus did best as well, pictures ended last with the video presentation in between. It shows how the presentation of some products can be improved by specific technology. 
H1c: The form of the presentation influences the attitudes towards the use of in-house technology.

The results obtained prove that VR technology facilitates the adoption of another technology.

\section{Conclusions}

VR technology is an emerging innovation that affects consumer behaviour and their perception of the products to which they are exposed to a great extent. Virtual Reality has been examined previously and found to have a positive impact on brand perceptions and purchase intentions thanks to an immersive brand experience (Van Kerrebroeck et al., 2017a). Virtual representations can give consumers an opportunity to better examine product items such as clothing through the applied technology (Verhagen, Vonkeman, \& van Dolen, 2016). The research, focused on the perception of marketing communication materials, brings some insights into how to conduct such activities in the era of modern technologies. It has also confirmed that VR technology can significantly facilitate presentation of certain market offers in the real estate business. The method applied in the research may become a common practice in this type of business industry in the future. It is possible imagine that one day, every offer on Gumtree or OLX - apart from the pictures of the apartment - will contain a VR presentation where the potential buyers / tenants are able to walk around the place in virtual reality just as they do now in the real world. The positive role of disruptive innovations in the context of marketing communication may result in an increase in companies' willingness to invest more in popularizing their offers through various forms of cooperation with VR software creators. Technological innovations have laid the grounds for a completely new field of communication and content delivery and an innovative approach to them can make the flow of information between different market entities much easier.

Marketing specialists can benefit from this research as well because it clearly shows that traditional paper advertising is the weakest channel of communicating an offer in comparison to more advanced methods (such as video or VR presentations), which proves the great importance of marketing virtualization. Marketing managers should pay more attention to the process of selection of technologies that are to be used in communicating marketing messages. As indicated in the research the use of modern technologies such as video and VR has a very positive impact on the perception of marketing message and translates into a much better reception of the message among potential customers, which means that these technologies can significantly support marketing strategies and sales - especially of equipment and solutions based precisely on such modern technologies. Further research should focus on conducting 
similar experiments for various types of product categories, the relationships between the reception of technology, the message, and the offer, and the purchase decisions along with the managerial implications of virtual reality usage in marketing communication strategies and tactics.

\section{Appendix 1. Questionnaire ${ }^{10}$}

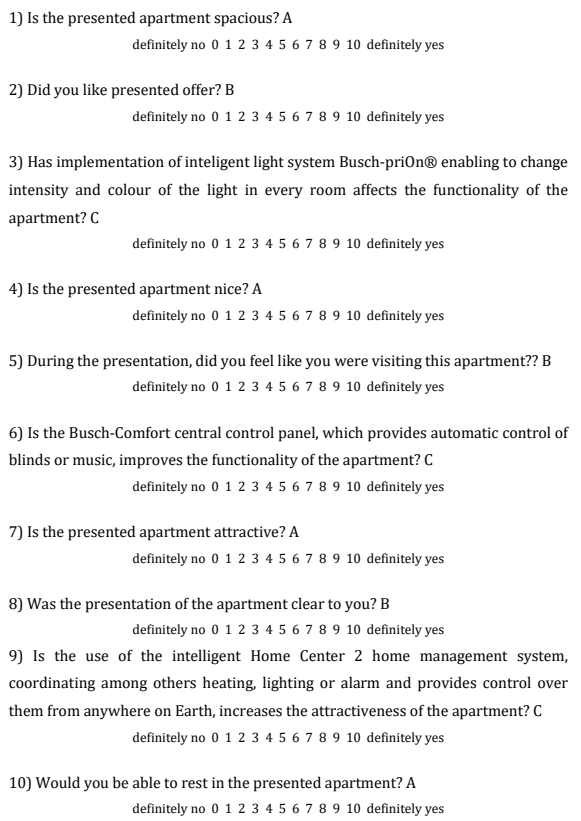

11) How realistically the presentation presented the apartment? B definitely not realistic 01123345678910 definitely realistic

12) Does the application of APA Vision BMS modern monitoring, allowing the preview of cameras from any device anywhere in the world, increases the security of housing? C

definitely no 0122345678910 definitely yes

13) Is the presented apartment cozy? A

definitely no 012345678910 definitely yes

14) Has the way of presenting the apartment underlined its qualities? B definitely no 0122345678910 definitely yes

15) Does the installation of the automatically locking blueMatic EAV lock in the entrance door, opened by a key, a chip in the key ring or a fingerprint reader, increases the security of the flat? C definitely no 012345678910 definitely yes

16) According to you what is the standard of the apartment presented? A definitely low 01223455678910 definitely high

17) To what extent presentation of the offer was pleasant for you? $B$ definitely not pleasant 01123345678910 definitely pleasant

18) Does the use of new technologies increase the attractiveness of the flat? $C$ definitely no 0123345678910 definitely yes

Age:

Sex: $\mathrm{w} / \mathrm{M}$

\section{References}

Adams, R. L. (2016, October 17). Five ways virtual reality will change the world. Forbes. Retrieved from https://www.forbes.com/sites/robertadams/2016/10/17/5-ways-virtual-reality-will-change-the-world/\#311e2cc2b018

Allameh, S. M., Esfahani, S. L., \& Nikbakht, M. (2017). Evaluating the effect of marketing innovation on green marketing strategies. International Journal of Scientific Management \& Development, 5(10), 464-473.

Barnes, S. (2016, November 3). Understanding virtual reality in marketing: nature, implications and potential. doi: https://dx.doi.org/10.2139/ssrn.2909100

${ }^{10}$ Items 1, 4, 7, 10, 13, 16 (A) relate to perception of the presented apartment; items 2, 5, 8, $11,14,17$ (B) relate to appraisal of the form of presentation; items 3, 6, 9, 12, 15, 18 (C) relate to attitude towards innovative technology in the presented apartment. 
Benefield, J. D., Rutherford, R. C., \& Allen, M. T. (2012). The effects of estate sales of residential real estate on price and marketing time. Journal of Real Estate Finance and Economics, 45(4), 965-981.

Biocca, F. (1992a). Virtual reality technology: a tutorial. Journal of Communication, 42(4), 23-72.

Biocca, F. (1992b). Communication within Virtual Reality: creating a space for research. Journal of Communication, Autumn, 42(4), 5-22.

Cheng, L. K., Chieng, M. H., \& Chieng, W. H. (2014). Measuring virtual experience in a three-dimensional virtual reality interactive simulator environment: a structural equation modelling approach. Virtual Reality, 18, 173-188.

Choi, Y. K., \& Taylor, C. R. (2014). How do 3-dimensional images promote products on the Internet?. Journal of Business Research, 67, 2164-2170.

Coates, G. (1992). Program from invisible site - a virtual shop, a multimedia performance. San Francisco, CA: Performance Works.

Cohen, J. (1988). Statistical power analysis for the behavioral sciences (2nd ed.). Hillsdale, NJ: Lawrence Erlbaum Associates.

Conover, W. J., \& Iman, R. L. (1981). Rank transformations as a bridge between parametric and nonparametric statistics. The American Statistician, 35(3), 124-129.

Cortina, J. M. (1993). What is coefficient alpha? An examination of theory and applications. Journal of Applied Psychology, 78(1), 98-104.

Gartner. (2016). Gartner's 2016 hype cycle for emerging technologies identifies three key trends that organizations must track to gain competitive advantage. Retrieved from https://www.gartner.com/newsroom/id/3412017

George, D., \& Mallery, P. (2003). SPSS for Windows step by step: a simple guide and reference 11.0 update (4th ed.). Boston, MA: Allyn \& Bacon.

Grigorovici, D. M. \& Constantin, C. D. (2004). Experiencing interactive advertising beyond rich media: impacts of ad type and presence on brand effectiveness in $3 \mathrm{D}$ gaming immersive virtual environments. Journal of Interactive Advertising, 5(1), 22-36.

Hair, J. F., Black, W. C., Babin, B. J., \& Anderson, R. E. (2010). Multivariate data analysis: a global perspective (7th ed.). Upper Saddle River, NJ: Pearson-Hall International.

Hajduk, G. (2016). Challenges for marketing communications in the digital age. In G. Mazurek \& J. Tkaczyk (Eds.), The impact of the digital world on management and marketing (pp. 183-195). Warszawa: Poltext.

Hassouneh, D., \& Brengman, M. (2015). Retailing in social virtual worlds: developing a typology of virtual store atmospherics. Journal of Electronic Commerce Research $16,218-241$.

Hopkins, C. D., Raymond, M. A., \& Mitra, A. (2004). Consumer responses to perceived telepresence in the online advertising environment: the moderating role of involvement. Marketing Theory, 4(1-2), 137-162.

Huang, Y. C., Backman, K. F., Backman, S. J., \& Chang, L. L. (2015). Exploring the implications of virtual reality technology in tourism marketing: an integrated research framework. International Journal of Tourism Research, 18(2), 116-128.

Jung, T., Dieck, M. C., Lee, H., \& Chung, N. (2016). Effects of virtual reality and augmented reality on visitor experiences in museum. In A. Inversini, \& R. Schegg (Eds.), Information and Communication Technologies in Tourism [E-Reader Version]. 
Retrieved from https://link.springer.com/book/10.1007\%2F978-3-319-282312\#page $=612$

Kaplan, A. \& Mazurek, G. (2018). Social media: state of the art and research agenda. In B. Mierzejewska, J. Jung, \& A., Albarran, (Ed.), 2nd handbook of media management and economics. London, United Kingdom: Routledge.

Keng, C., \& Lin, H. (2006). Impact of telepresence levels on Internet advertising effects. Cyberpsychology and Behavior, 9(1), 82-94.

Kirk, R. E. (2013). Experimental design: procedures for the behavioural sciences (4th ed.). London: Sage Publications.

La Valle, S. M. (2017). Virtual Reality. Cambridge: Cambridge University Press.

Lessiter, J., Freeman, J., Keogh, E., \& Davidoff, J. (2001). A cross-media presence questionnaire: the ITC-sense of presence inventory. Presence: Teleoperators and Virtual Environments, 10(3), 282-297.

Mandelbaum, A. (2015, August 17). How companies are marketing with virtual reality [Web log post]. Retrieved from http://www.chiefmarketer.com/companiesmarketing-virtual-reality/

Mazurek, G. (2011a). Informacja w wirtualnym środowisku a rozwój społeczeństwa informacyjnego. Zeszyty Naukowe Uniwersytetu Szczecińskiego. Ekonomiczne Problemy Ustug, 186-194.

Mazurek, G. (2011b). Virtualization of marketing - conceptual model. In C. Wang (Ed.), Proceedings of the International Conference on Marketing Studies (pp. 109-113). Academy of Taiwan, Kuala Lumpur, Malaysia.

Mazurek, G. (2012). Virtualization of marketing. Contemporary Management Research, 8(3), 195-204.

Mazuryk, T., \& Gervautz, M. (1996). Virtual Reality: history, applications, technology and future, technical report. Vienna: Institute of Computer Graphics, Vienna University of Technology.

Saunders, M., Lewis, P., \& Thornhill, A. (2009). Research method for business students (5th ed.). Prentice Hall, London: Pitman.

Sax, L. (2006). Why gender matters: what parents and teachers need to know about the emerging science of sex differences. Westminster, MD: Broadway Books.

Scatena, S., Russo, G.N., Riva, G. (2016). Virtual Reality vs television vs web exposure: the impact on brand experience. A preliminary study. Annual Review of Cybertherapy and Telemedicine, 14, 211-214.

Schroeder, J. A. (2010). Sex and gender in sensation and perception. In J. C. Chrisler, D. R. McCreary (Eds.), Handbook of gender research in psychology. Vol. 1: Gender research in general and experimental psychology (pp. 235-258). Boston: Springer.

Scott, J. (2016). Virtual Reality: content marketing's next big trend [Web log post]. Retrieved from https://www.econsultancy.com/blog/68401-virtual-reality-contentmarketing-s-next-big-trend

Serrano, B., Botella, C., Baños, R. M., \& Alcañiz, M. (2013). Using virtual reality and mood-induction procedures to test products with consumers of ceramic tiles. Computers in Human Behavior, 29(3), 648-653.

Sherman, W. R. (2003). Understanding Virtual Reality. Burlington, MA: Morgan Kaufman Publishers. 
Steuer, J. (1992). Defining Virtual Reality: dimensions determining telepresence. Journal of Communication, 42(4), 73-93.

The Farm 51. (2015). VR market report. Retrieved from http://thefarm51.com/ripress/ VR_market_report_2015_The_Farm51.pdf

Tomczak M., \& Tomczak, E. (2014). The need to report effect size estimates revisited. An overview of some recommended measures of effect size. Trends in Sport Sciences, 1(21), 19-25.

Van Kerrebroeck, H., Brengman, M., \& Willems, K. (2017a). Escaping the crowd: an experimental study on the impact of a Virtual Reality experience in a shopping mall. Computers in Human Behavior, 77, 437-450.

Van Kerrebroeck, H., Brengman, M., \& Willems, K. (2017b). When brands come to life: experimental research on the vividness effect of Virtual Reality in transformational marketing communications. Virtual Reality, 21(4), 177-191.

Verhagen, T., Vonkeman, C., \& van Dolen, W. (2016). Making online products more tangible: the effect of product presentation formats on product evaluations. Cyberpsychology, Behavior, and Social Networking, 19(7), 460-464.

Yin, M., Cicchirillo, V., \& Drumwright, M. (2012). The impact of stereoscopic threedimensional (3-D) advertising. The role of presence in enhancing advertising effectiveness. Journal of Advertising, 41, 113-128.

Zheng, L. (2016, March 18). Alibaba spreads its wings into VR sector [Web log post]. Retrieved from http://www.chinadaily.com.cn/business/tech/2016-03/18/content _23945440.htm 